\title{
LINE PROFILE VARIATIONS IN \\ WR + COLLAPSED COMPANION SYSTEMS
}

\author{
GLORIA KOENIGSBERGER \\ Instituto de Astronomía, UNAM, Mexico
}

\begin{abstract}
The existence of Wolf-Rayet binary systems containing a collapsed companion is predicted on evolutionary grounds. However, because of the absence of strong X-ray emission and the difficulty in obtaining consistent periodicities from the observed variations, none of the "classical" WR stars has been shown to be a WR+cc system. A discussion is presented of the line-profile variations which are predicted to occur on orbital timescales due to the interaction of the WR wind with the cc. We note the importance of numerical simulations of the flow structure of the WR wind in the vicinity of a collapsed companion for deriving diagnostic tools for the presence of a cc in a WR system.
\end{abstract}

Key words: : stars: Wolf-Rayet - binaries - line profiles - individual: HD 50896

\section{Introduction}

The existence of Wolf-Rayet (WR) binaries containing a collapsed companion (cc) was first predicted by van den Heuvel \& Heise (1972) on the basis of theoretical models of the evolution of massive stars in close binary systems. In these models a system consisting initially of two massive O-type stars with similar masses evolves into an $\mathrm{O}+\mathrm{WR}$ system, after which the WR star undergoes a supernova event leaving behind a remnant collapsed object; i.e., a neutron star or a black hole. These systems are observed to exist and constitute the class of massive binary X-ray sources with X-ray luminosities of $10^{36}$ to $10^{38} \mathrm{erg} \mathrm{s}^{-1}$. Subsequent evolution of the $\mathrm{O}$ star in such systems is expected to produce a WR+cc system.

From an observational standpoint, there are a number of WR stars which exhibit photometric, polarization and line profile variability which could potentially be attributed to the presence of a cc. The bright WN5 star, HD 50896 (WR6), was the first WR to be proposed to have a collapsed companion (Koenigsberger 1978; Firmani et al. 1980), based on the discovery of periodic emission-line profile variations, in addition to its large distance from the orbital plane, and its association with the ring nebula S308. Other WRs were soon after found to present line-profile variations and were also proposed to be WR+cc systems (see reviews by Moffat 1982; Cherepashchuk \& Aslanov 1984). Given that WR stars possess strong stellar winds, the interaction of the wind with the cc is predicted to occur via a shock cone and the accretion of the shocked gas by the cc is expected to generate X-ray emission. However, the levels of X-ray emission which have been observed from the "classical" WRs ( $c f$. those in the van der Hucht et al. 1981 catalogue), are several orders of magnitude below the levels which would be predicted from a standard wind accretion model (Stevens \& Willis 1988). In 
the case of HD 50896, Stevens \& Willis (1988) conclude that if HD 50896 is indeed a WR+cc system, then the most likely companion would be a nonaccreting neutron star. Such a model has been suggested by Stella, White \& Rosner (1986), in a scenario in which the neutron star is spun up by the accretion of angular momentum until centrifugal inhibition of accretion occurs and the system is X-ray quiet. Alternative explanations for the variabiity in HD 50896 and other WRs with similar characteristics include the presence of a disk (Rumpl 1980; Schulte-Ladbeck et al. 1991), rotating jet-like structures (Matthews et al. 1992), and intrinsic wind instabilities (St-Louis et al. 1993). Thus, observational evidence for the existence of WR+cc systems is as yet inconclusive.

The discovery of a WR-like IR spectrum in the strong X-ray source Cyg X-3 (van Kerkwijk et al. 1992, these proceedings) may be providing the evidence needed to prove the existence of WR+cc systems. However, given the very short orbital period (4.8 hours) it also raises questions regarding the accretion flow and the degree of distortion of the WR wind structure by the emitted X-rays.

Because of the uncertainties surrounding the existence of WR+cc systems, it is of interest to develop diagnostics which can provide indications of the presence of a cc in a WR binary system. The crucial element in such an analysis is the interaction of the WR wind with the cc, since it is through this interaction that observational features will arise.

\section{The morphology of the flow in the vicinity of the companion}

A collapsed companion in orbit within the stellar wind of a WR star will affect the dynamics of the flow in several ways. The gravitational field will deflect the wind particles from their radial trajectories producing a bowshock, behind which gas is accreted onto the cc. This accretion gives rise to $\mathrm{X}$-ray emission (Davidson \& Ostriker 1973). The X-ray emission creates a photo-ionized region around the cc (Hatchett \& McCray 1977; Kallman \& McCray 1982), and affects the dynamics of the flow near the cc, both through radiation pressure (Tarter \& McKee 1973; Hatchett, Buff \& McCray 1976) and through heating (Buff \& McCray 1974).

Calculations of the flow structure which include the effects of gravity, Xray ionization, heating and cooling have only recently become available, and apply to the case in which the wind-shedding star is an O-type star. The major difference between WR and $\mathrm{O}$-stars are the chemical composition of the winds, with WRs' being highly enriched in He and $\mathrm{N}$, or He and $\mathrm{C}$ with respect to $\mathrm{O}$-stars and in the mass-loss rates, which in the WRs are a factor an order of magnitude larger than in $\mathrm{O}$-stars. Although these differences are important, we consider the calculations for the $\mathrm{O}+\mathrm{cc}$ systems to be very illustrative. In Figure 1 we reproduce the result of a $2 \mathrm{D}$ numerical simulation 


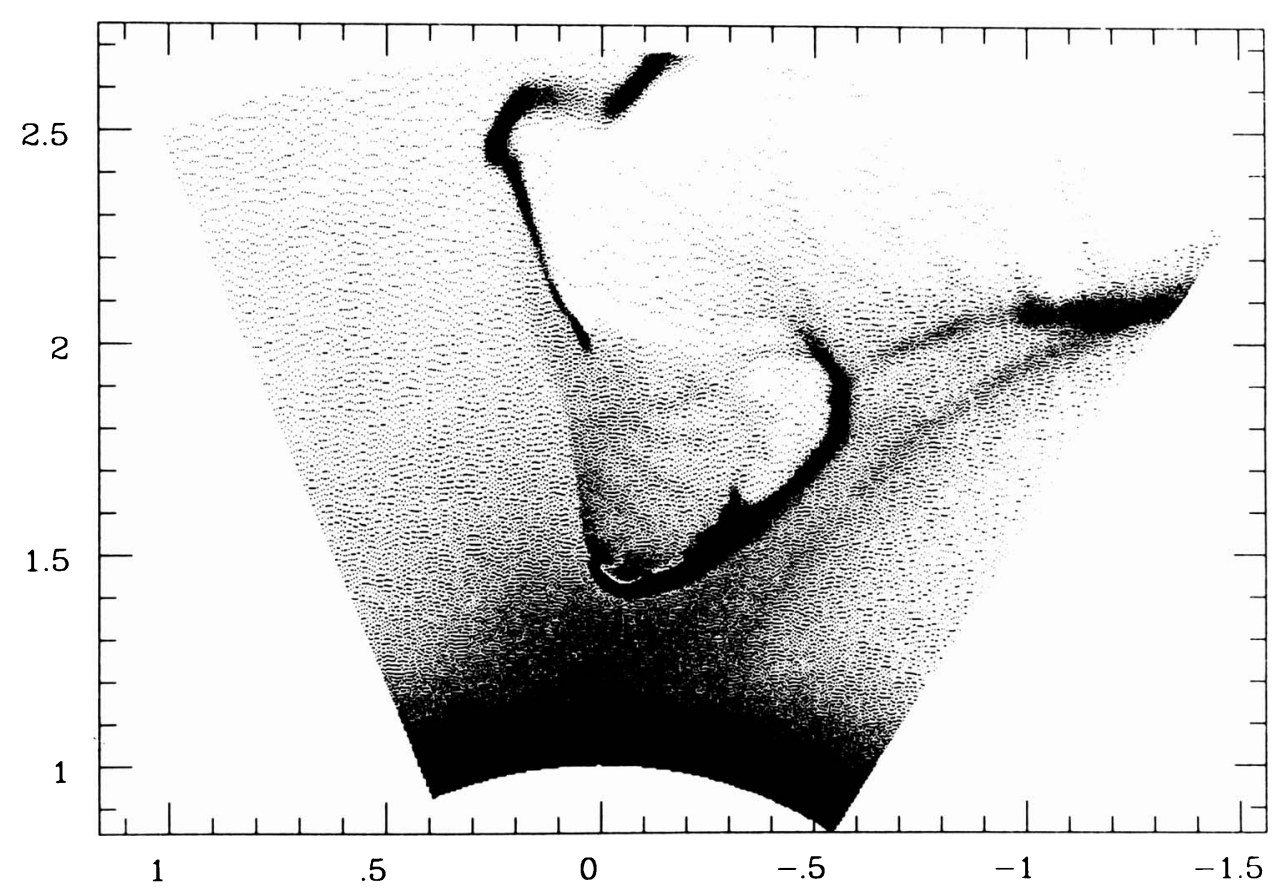

Fig. 1. Numerical simulation of the accretion wake in a MXBS such as Vela X-1. The density scale goes from $10^{9} \mathrm{~cm}^{-3}$ (white) to $10^{13} \mathrm{~cm}^{-3}$ (black). The physical dimensions are in units of $2.5 \times 10^{12} \mathrm{~cm}$ (taken from Figure 9 of Blondin et al. 1990).

of the accretion flow for inferred parameters of the binary X-ray source Vela X-1 (4U 0900-40) by Blondin et al. (1990), in which the complicated structure of this flow is evident. In this calculation, within the zone that is ionized by the $\mathrm{X}$-rays, the wind is no longer accelerated by the radiation flux of the $\mathrm{O}$-star primary. The resulting decrease in wind velocity at the compact object leads to a photo-ionization wake trailing behind the Strömgren zone where the slower wind is overtaken by the unaffected wind. The compression of the gas in the accretion wake can produce densities approaching 100 times the density in the undisturbed wind. It is also important to note that although the accretion radius is only $7 \times 10^{10} \mathrm{~cm}$, the dimensions of the perturbed wind surrounding the cc are much larger, extending out to at least $2.5 \times 10^{12} \mathrm{~cm}$ from the cc.

In addition to the complex nature of the flow, the numerical simulations have revealed that in many cases the two-dimensional flow does not reach a steady state, but rather, the wake shock-cone oscillates from side to side. These instabilities were first discovered by Matsuda et al. (1987) and 
Taam \& Fryxell (1988) in calculations of flow structures in the presence of asymmetries in the incident flow, and were termed the "flip-flop" instability. The flip-flop motion results in the temporary formation of an accretion disk which can circulate in either sense about the compact companion (assumed to be a neutron star in these calculations). The flip-flop instability also appears in the calculations of Blondin et al. (1990), where for X-ray sources of moderate strength $\left(L_{x} \approx 10^{36} \mathrm{erg} \mathrm{s}^{-1}\right)$, the time-dependent accretion wake contains dense filaments of compressed gas. The temporal behavior of the simulations shows a well-defined time-scale, which can be as short as $10^{3}$ sec, but no coherence over more than a few oscillations. At higher X-ray luminosities $\left(L_{x} \approx 10^{37} \mathrm{erg} \mathrm{s}^{-1}\right)$, Compton cooling near the compact object keeps the flow roughly iso-thermal, and the accretion is more nearly radial. In this case, the accretion flow becomes steady and the column density in the accretion wake is relatively small.

The flip-flop instability does not appear to be as violent when the hydrodynamical calculations are performed in 3D (Ishii et al. 1993; Livio et al. 1991), rather than in 2D, although the 3D calculations have not been performed with the same (high) resolution as have the 2D calculations.

It is interesting to note the suggestion of Ishii et al. (1993) in the sense that binary systems in which significant mass is lost through episodic equatorial mass-shedding events (such as in Be stars), approximate the conditions of $2 \mathrm{D}$ calculations, and that strong flip-flop instabilities are thus expected to appear. If some of the WR stars have disk-like structures as seems to be the case (Cassinelli, these proceedings; Schulte-Ladbeck, these proceedings), it is tempting to suggest that the flip-flop instabilities would arise associated to a cc in orbit about such stars. These instabilities would lead to variability in the spectrum on timescales shorter than the orbital period.

\section{Line profile variations}

One of the observational consequence of the X-ray heating of the dense WR wind was suggested by Hatchett \& McCray (1977). As the X-ray source passes between the normal star's continuum emitting region and the observer, the altered ionization structure of the wind can produce a deficit of absorbing/scattering ions involved in the production of the absorption components in P-Cygni line profiles. Thus, when the collapsed object is "in front" of the companion, one expects to observe weaker P-Cygni absorption components. These variations occur periodically on orbital time-scales, and have been detected in systems such as Vela X-1 (Dupree et al. 1978) and Cyg X-1 (Hammerschlag-Hensberge 1980), and has been termed the "Hatchett-McCray effect". The search for a Hatchett-McCray effect in the UV spectra of WR stars has not been as conclusive. Smith et al. (1985), and St-Louis et al. (1989 and 1993) have found variations in the P-Cygni 
absorption components in the UV spectra of HD 96548, HD 192163 and HD 50896, respectively. But because of the absence of a clear periodicity, or to periodicity different from the reported possible binary periods, it was difficult to interpret the variations in terms of the Hatchett-McCray effect, and instead, they were attributed to intrinsic WR stellar wind variations.

Koenigsberger (1978) and Firmani et al. (1980) reported phase-dependent changes in the P-Cygni absorption components of the N v $4603,4619 \AA$ doublet in HD 50896. The changes, consisting of the periodic weakening of the P-Cygni absorption components, are consistent with the Hatchett-McCray effect and are still observed in recent data of HD 50896 (Cardona et al. in preparation). Thus, there is some evidence for the presence of a HatchettMcCray effect in HD 50896, if only in the weak N v 4603,4619 $\AA$ lines.

Modeling efforts to produce spectroscopic diagnostics for the presence of a cc in an intense stellar wind based on numerical simulations of line profile variations have been scarce and based on simplified assumptions. In the simplest scenario, one can postulate the presence of emission lines associated with the cc, and moving with the same orbital velocity. The combined spectrum of the WR and the cc will undergo orbital phase-dependent variations due to: (a) the motion of the cc in its orbit, and (b) the radiative transfer of its emission through the WR wind.

A discussion of the various mechanisms which can produce phase-dependent line-profile variations in WR binary systems was given by Koenigsberger \& Auer (1992). They presented synthetic profiles to illustrate the effects due to a physical eclipse and the contribution by line emission from a companion. In the case in which the companion has an associated emission line it is superposed on the broader WR emission line and the combined profiles may present asymmetries and multiple maxima, depending on the orbital phase. In Figure 2 we illustrate the nature of these variations. The parameters and the method for carrying out the numerical simulations of the emergent line profiles are described in Auer \& Koenigsberger (1994).

The synthetic profiles have qualitative similarities with the He II $4686 \AA$ profiles in HD 50896 (see for example Figure 1 of Firmani et al. 1980), although a phase-to-phase comparison indicates important differences. If HD 50896 is indeed a WR+cc system, differences between the observed and synthetic He II line-profiles may be attributable to the (current) limitations in the numerical simulations. These limitations are: (1) the assumption of a constant source function throughout the WR wind line-emitting region; (2) the assumption of spherical symmetry of the WR wind, i.e., neglect of the effects that the companion has on the dynamics and ionization structure of the wind; and (3) the assumption of a Gaussian line-profile associated with the companion and undergoing velocity displacements according to the orbital motion of the cc. Given the structure of the flow surrounding the cc as shown in Figure 1, these assumptions are important limitations and should 

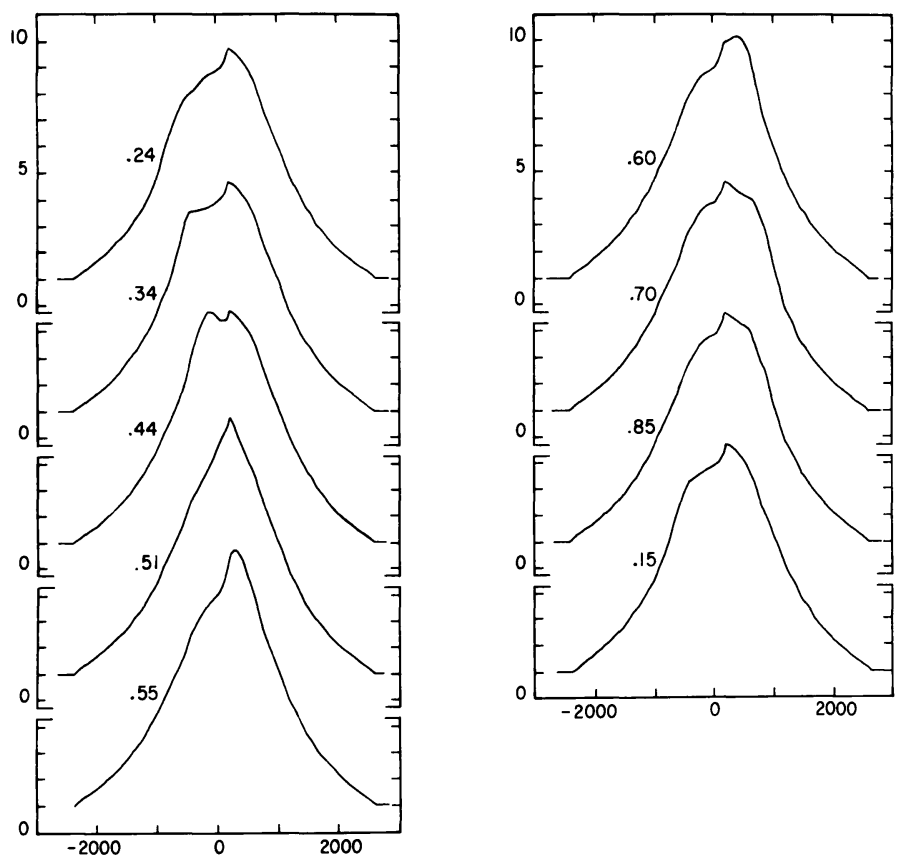

Fig. 2. Numerical simulations of emission line profiles of a WR+cc binary system in a simplified model as a function of orbital phase. Phase 0.50 corresponds to cc "in front". The companion's emission line is assumed to originate close to the cc and to share in its orbital motion. The orbit lies within the WR line-emitting region $\left(R_{\text {orb }} / R_{*}=15\right.$; $\left.R_{e m} / R_{*}=30\right)$. The WR wind is assumed to accelerate linearly out to $29 R_{*}$ and the opacity factor used is $\chi=4 \times 10^{3}$. There is little change in the profiles between phases 0.85 and 0.15 due to the large optical depth of the WR wind.

be modified in future calculations. We note however, that the underlying shape of the observed line profiles are very similar to the shape produced by these simulations, in which a very extended acceleration region in the WR wind is assumed. An extended acceleration region in WR winds has been suggested previously (Koenigsberger 1988, 1990) based on semi-empirical considerations, and now is starting to have a physical basis (Lucy \& Abbott 1993; Springmann 1994).

\section{Conclusions}

The numerical (2D) simulations of the flow structure of a wind in the vicinity of a collapsed companion reveal complex morphologies and the presence of instabilities which can give rise to line-profile variability on time-scales which are shorter than the orbital period. These variations would be superposed 
upon the orbital phase-dependent variations, and would thus contribute toward the difficulty in establishing unique periods for observed variations in WR+cc candidates. Numerical simulations in $3 \mathrm{D}$, as well as numerical simulations applicable to systems in which the primary is a WR star, are urgently needed. These would facilitate more realistic calculations of the expected line-profile variations in $\mathrm{WR}+\mathrm{cc}$ systems, and would allow a determination of the role that flow-structure instabilities may play in producing short timescale variability in WR systems. Diagnostic tools may then be derived in order to determine whether any of the WR+cc candidates do indeed contain a collapsed companion.

It is important to note that the presence of a low mass, non-X-ray emitting companion in a WR wind is also expected to give rise to density enhancements near the companion. Thus, discriminating between the presence of an X-ray "quiet" collapsed companion and a normal low-mass companion in WR systems will require critical application of the diagnostic tools.

\section{Acknowledgements}

I wish to express my gratitude to Jorge Cantó for many fruitful discussions, to Mario Livio for helpful suggestions and to Gabriela Canalizo for much assistance during the preparation of this paper. I thank John Blondin for authorizing the reproduction of Figure 9 from Blondin et al. (1990), and Alberto García and Juan Carlos Yustis for the figures, and Bertha Ma. Hernández for production of this text. Travel was supported by CONACYT grant 1160-E and UNAM/DGAPA grant IN104591.

\section{References}

Auer, L.H., Koenigsberger, G 1994, $A p J$ in press

Blondin, J.M., Kallman, T.R., Fryxell, B.A., Taam, R.E. 1990, ApJ 356, 591

Buff, J. and McCray, R.A. 1974, ApJ 189, 147

Cherepashchuk, A.M., Aslanov, A.A. 1984, Ap Space Sci. 102, 97

Davidson, K., Ostriker, J.P. 1973, $A p J$ 179, 588

Dupree, A., Willis, A.J., van den Heuvel, E.P.J. et al. 1978, Nature 275, 400

Firmani, C., Koenigsberger, G., Bisiacchi, G.F., Moffat, A.F.J., Isserstedt, J.1980, ApJ 239, 607

Hammerschlag-Hensberge, G. 1980, in: Proc. 2nd Eur. IUE Conf., ESA SP-157, p. lix

Hatchett, S.P., McCray, R. 1977, ApJ 211, 552

Hatchett, S.P., Buff, J. and McCray, R.A. 1976, $A p J$ 206, 847

van den Heuvel, E.P.J., Heise, J. 1972, Nature 239, 67

van der Hucht, K.A., Conti, P.S., Lundström, I., Stenholm, B. 1981, Sp. Sci. Rev. 28, 227

Ishii, T., Matsuda, T., Shima, E., Livio, M., Anzer, U., Börner, G. 1993, ApJ 404, 706

Kallman, T.R., McCray, R. 1982, ApJ Suppl. 50, 263

van Kerkwijk, M.H., Charles, P.A., Geballe, T.R., King, D.L., Miley, G.K., Molnar, L.A., van den Heuvel, E.P.J., van der Klis, M., van Paradijs, J. 1992, Nature 355, 703

Koenigsberger, G. 1978, Tesis de Lic., Fac. Ciencias, UNAM

Koenigsberger, G. 1988, Rev. Mexicana Astron. Astrof. 16, 85

Koenigsberger, G. 1990, $A \mathscr{B} A$ 235, 282. 
Koenigsberger, G., Auer, L.H. 1992, in: L. Drissen, L., C. Leitherer, \& Nota, A. (eds.), Nonisotropic and Variable Outflows from Stars, ASP Conf. Series 22, 239.

Livio, M., Soker, N., Matsuda, T., Anzer, U. 1991, MNRAS 253, 633

Lucy, L.B., Abbott, D.C. 1993, ApJ 405, 738

Matthews, J.M., St-Louis, N., Moffat, A.F.J., Drissen, L., Koenigsberger, G., Cardona, O., Niemela, V.S. 1992, in: L. Drissen, L., C. Leitherer, \& Nota, A. (eds.), Nonisotropic and Variable Outflows from Stars, ASP Conf. Series 22, 130

Matsuda, T., Inoue, M., Sawada, K. 1987, MNRAS 226, 785

Moffat, A.F.J. 1982, in: C.W.H. de Loore \& A. J. Willis (eds.), Wolf-Rayet Stars, Observations, Physics, Evolution, Proc. IAU Symp. No. 99 (Dordrecht: Reidel), p. 263

Rumpl, W.M. 1980, ApJ 241, 1055

Schulte-Ladbeck, R.E., Nordsieck, K.H., Taylor, M., Nook, M.A., Bjorkman, K.S., Magalhães A.M., Anderson, C.M. 1991, $A p J$ 382, 301

Smith, L.J., Lloyd, C. Walker, E.N. 1985, $A \& A$ 146, 307

Springmann, U. 1994, $A \& A$ in press

St-Louis N., Smith, L.J., Stevens, I.R., Willis, A.J., Garmany, C.D., Conti, P.S. 1989, $A \& A$ 226, 249

St-Louis, N., Howarth, I.D., Willis, A.J., Stickland, D.J., Smith, L.J., Conti, P.S., Garmany, C.D. 1993, ApJ 267, 447

Stella, L., White, N.E., Rosner, R. 1986, ApJ 308, 669

Stevens, I.R., Willis, A.J. 1988, MNRAS 234, 783

Taam, R.E., Fryxell, B.A. 1988, ApJ (Letters) 327, L73

Tarter, L.B., McKee, C.F. 1973, ApJ (Letters) 186, L63

\section{DISCUSSION:}

van Kerkwijk: Could the companion be a solar-type star instead of a compact object? From evolutionary considerations, which systems should exist (O stars with low-mass companions have been observed) and this would solve the problem that no strong X-ray emission is observed.

Koenigsberger: If the line profile variations in Hell 4686 in HD 50896 can be modelled in detail with the assumption of secondary emission arising in the shock cone, then the answer is "Yes". All we need is enhanced-density regions, which can be found in the shock cone.

Antokhin: I think the problem of your model is that the NS itself does not radiate optical emission lines. To have lines, you should have accretion. This means you have X-ray radiation. As a consequence, additional flux in emission line will arise from a large ionized volume. Thus, you won't have narrow emission features, but instead very broad and shallow ones. They will be hardly detected. I think we can only hope to see any effects in absorption parts of resonance UV lines.

Koenigsberger: The density enhancements in the flow calculations of Blondin et al. (1990) for Vela X-1 are $\sim 100$ times the unperturbed O-star wind. HeII emission associated with the companion could arise in these filamentary structures. The velocity distribution for these filaments is what we have not included in the calculation as yet. 
Kaper: If we assume that Cyg X-3 is a WR/NS binary that has a slightly tighter orbit than the proposed $0.4 \mathrm{~d}$ orbit in $\mathrm{HD} 50896$, why do we not see any orbital modulation with this period in UV line profiles (cf St-Louis et al.) due to the (huge) X-ray luminosity?

Koenigsberger: The period in $\mathrm{HD} 50896$ is 3.76 days. The $\sim 0.43$ day period wyhich was found by Cordona et al. might be associated with "flip-flop" instabilities, if it is confirmed, or some other type of oscillation.

Maeder: Is the number of binary systems of the type (WR + compact) consistent with theoretical number expectations?

van Kerkwijk: Given the large uncertainties about the spiral-in process, it is hard to predict the number of $W R+C$ systems from the number of progenitors. According to Matthew Bailes, one expects only one WR+C based on the number of observed descendant systems (doubleneutron star binaries like the Hulse-Taylor pulsar).

Lindsey Smith: A totally naive question. Why - if this is a compact accreting object - do we not, from time to time, see a thermonuclear runaway, as in a nova? [And, if you answer as you did at the time - may I add ... then why do we not see, from time to time, the Gamma rays?] van Kerkwijk: For a neutron-star companion, I do not think thermonuclear runaways are expected, since for these systems the accretion rate should be very high, while bursts - associated with thermonuclear runaways - are observed only in low-mass X-ray binaries where the accretion rate is relatively low Also among these, none show X-ray pulsations, indicating that the magnetic field is low. For a companion to a Wolf-Rayet star, appreciable magnetic field are expected. [ Furthermore, the lack of hydrogen in the accreted matter would make thermonuclear runaway a much less energetic event.]

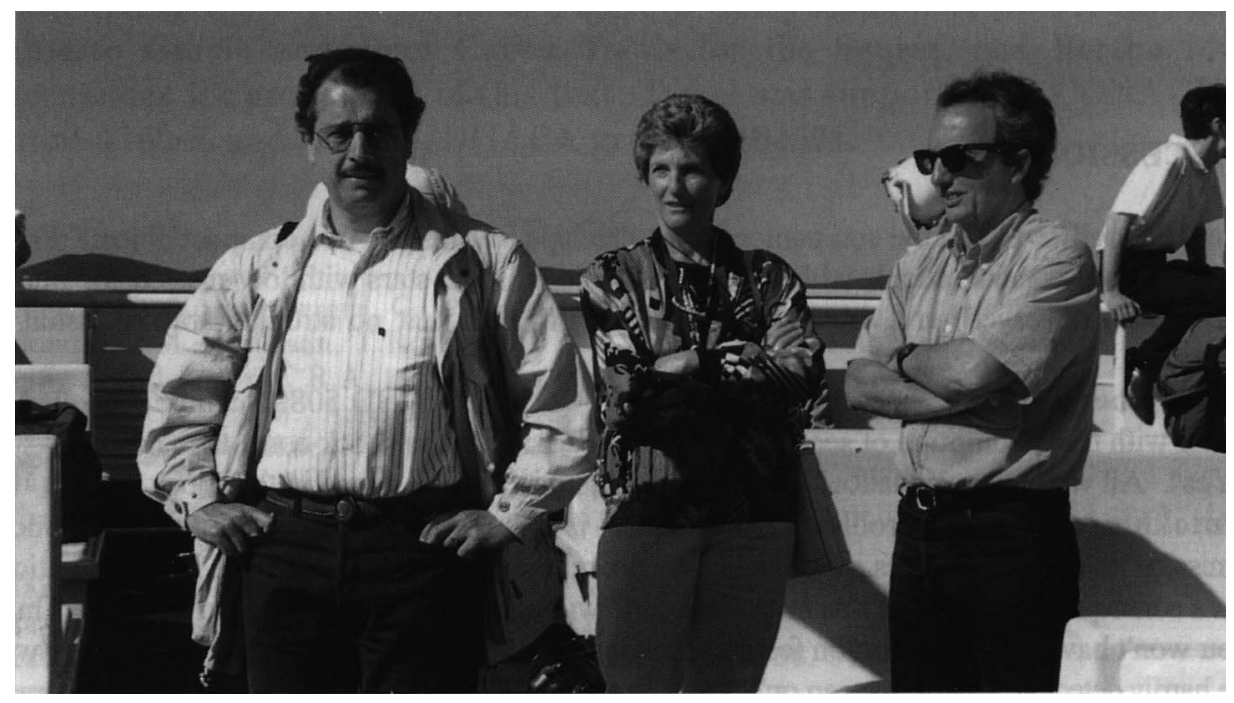

Breysacher, Mrs. Breysacher, Azzopardi 